\title{
RESPONSIBILITY ASSIGNMENT IN SYSTEMS ENGINEERING
}

\author{
Iris Gräßler, Henrik Thiele, Benedikt Grewe, Michael Hieb \\ Paderborn University, Germany \\ henrik.thiele@hni.uni-paderborn.de
}

\begin{abstract}
The constantly increasing system complexity leads to various challenges in established companies, which can be controlled by using systems engineering processes. INCOSE defines explicit processes with concrete inputs and outputs (artifacts) for this purpose. Systems Engineering roles can be used to organize the tasks of the processes within the company. In this work, the responsibilities of the systems engineering roles on the process artifacts are evaluated by means of the RACI scheme and examined by means of cluster analysis. The work steps are accompanied by discussions and results of a systems engineering transformation project with a large German automotive OEM. The goal of the study is to identify the minimum size of a systems engineering team and prioritize the systems engineering roles. As a result, the (sometimes contradictory) requirements from the methodological perspective of systems engineering and the organizational perspective of project management become manageable in the control and execution of systems engineering projects.
\end{abstract}




\begin{tabular}{|c|c|c|c|c|c|c|c|c|c|c|c|c|c|c|c|}
\hline Input/Output & $\begin{array}{c}\dot{00} \\
\dot{=} \\
\dot{0} \\
\dot{E} \\
\dot{\Sigma}\end{array}$ & 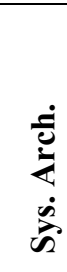 & $\begin{array}{l}\dot{\dot{\vec{b}}} \\
\sum^{0} \\
\dot{\Xi} \\
\dot{\vec{\Omega}} \\
\dot{\vec{n}}\end{array}$ & 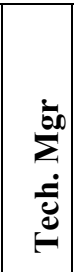 & 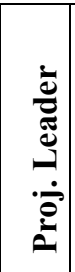 & 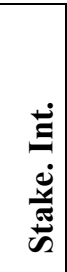 & 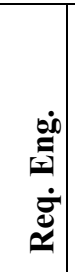 & 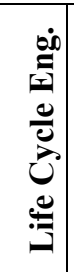 & 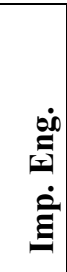 & 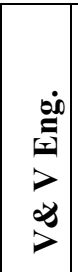 & 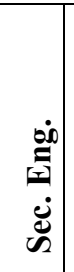 & 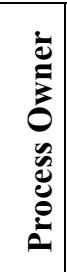 & 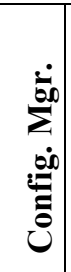 & $\begin{array}{c}\dot{\dot{g}} \\
\sum_{0} \\
\dot{\Xi} \\
\dot{\Xi} \\
\dot{\Xi}\end{array}$ & 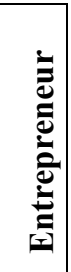 \\
\hline $\begin{array}{l}\text { Accepted system or system } \\
\text { element }\end{array}$ & $\mathrm{C}$ & $\mathrm{C}$ & $\mathrm{C}$ & $\mathrm{C}$ & $\begin{array}{l}\mathrm{R} \\
\mathrm{A}\end{array}$ & $\mathrm{C}$ & $\mathrm{C}$ & $\mathrm{C}$ & C & $\mathrm{C}$ & $\mathrm{C}$ & $\mathrm{C}$ & $\mathrm{C}$ & $\mathrm{C}$ & I \\
\hline Acquired system & & $\mathrm{C}$ & $\begin{array}{l}\mathrm{R} \\
\mathrm{A}\end{array}$ & $\mathrm{C}$ & & & $\mathrm{C}$ & & & & & & $\mathrm{C}$ & $\mathrm{C}$ & \\
\hline Acquisition agreement & $\mathrm{C}$ & & $\mathrm{C}$ & & A & & & & $\mathrm{C}$ & & & & & & $\mathrm{R}$ \\
\hline Acquisition need & $\mathrm{R}$ & $\mathrm{C}$ & $\mathrm{C}$ & & $\mathrm{A}$ & & & & $\mathrm{C}$ & & $\mathrm{I}$ & & $\mathrm{C}$ & $\mathrm{C}$ & $\mathrm{I}$ \\
\hline Acquisition payment & & & & & $\mathrm{C}$ & & & & & & & & & & $\begin{array}{l}\mathrm{R} \\
\mathrm{A}\end{array}$ \\
\hline Acquisition record & $\mathrm{C}$ & $\mathrm{C}$ & $\begin{array}{l}\mathrm{R} \\
\mathrm{A}\end{array}$ & $\mathrm{C}$ & & & I & & $\mathrm{C}$ & & & & $\mathrm{C}$ & & \\
\hline Acquisition reply & $\mathrm{C}$ & $\mathrm{I}$ & $\begin{array}{l}\mathrm{R} \\
\mathrm{A}\end{array}$ & I & $\mathrm{I}$ & & & & $\mathrm{C}$ & & & & $\mathrm{C}$ & & \\
\hline Acquisition report & I & I & $\begin{array}{l}\mathrm{R} \\
\mathrm{A}\end{array}$ & I & I & & & & I & & & & I & & \\
\hline Acquisition strategy & & & $\mathrm{R}$ & $\mathrm{C}$ & A & & & & & & & & & & $\mathrm{C}$ \\
\hline Agreements & & & & $\mathrm{C}$ & $\begin{array}{l}\mathrm{R} \\
\mathrm{A}\end{array}$ & & & & & & & & & & $\mathrm{C}$ \\
\hline Alternative solution classes & $\mathrm{C}$ & $\begin{array}{l}\mathrm{R} \\
\mathrm{A}\end{array}$ & & $\mathrm{C}$ & & $\mathrm{C}$ & $\mathrm{C}$ & & & & & & & & $\mathrm{I}$ \\
\hline Analysis situations & & $\mathrm{C}$ & $\mathrm{C}$ & $\begin{array}{l}\mathrm{R} \\
\mathrm{A}\end{array}$ & I & & $\mathrm{C}$ & & & & & & & $\mathrm{C}$ & \\
\hline Applicable laws and regulations & & & & & A & & $\mathrm{C}$ & & & $\mathrm{R}$ & $\mathrm{C}$ & & $\mathrm{C}$ & & \\
\hline Architecture definition record & & $\begin{array}{l}\mathrm{R} \\
\mathrm{A}\end{array}$ & & $\mathrm{C}$ & & & $\mathrm{C}$ & & & & & & & & \\
\hline Architecture definition strategy & & $\begin{array}{l}\mathrm{R} \\
\mathrm{A}\end{array}$ & & $\mathrm{C}$ & $\mathrm{C}$ & & $\mathrm{C}$ & & & & & & & & \\
\hline Architecture traceability & & $\begin{array}{l}\mathrm{R} \\
\mathrm{A}\end{array}$ & & $\mathrm{C}$ & & & $\mathrm{C}$ & & & & & & & & \\
\hline $\begin{array}{l}\text { Business or mission analysis } \\
\text { record }\end{array}$ & & & & & $\begin{array}{l}\mathrm{R} \\
\mathrm{A}\end{array}$ & & & & & & & & & & $\mathrm{C}$ \\
\hline $\begin{array}{l}\text { Business or mission analysis } \\
\text { strategy }\end{array}$ & & & & & $\begin{array}{l}\mathrm{R} \\
\mathrm{A} \\
\end{array}$ & & & & & & & & & & $\mathrm{C}$ \\
\hline Business requirements & & & & & $\mathrm{C}$ & & $\begin{array}{l}\mathrm{R} \\
\mathrm{A}\end{array}$ & & & & & & & & $\mathrm{I}$ \\
\hline Business requirements traceability & & & & & $\mathrm{C}$ & & $\begin{array}{l}\mathrm{R} \\
\mathrm{A}\end{array}$ & & & & & & & & $\mathrm{I}$ \\
\hline Candidate configuration items & & $\mathrm{C}$ & & $\mathrm{C}$ & & & & & & & & & $\begin{array}{l}\mathrm{R} \\
\mathrm{A}\end{array}$ & & \\
\hline Candidate information items & & $\mathrm{C}$ & & $\mathrm{C}$ & & & & & & & & & & $\begin{array}{l}\mathrm{R} \\
\mathrm{A}\end{array}$ & \\
\hline Candidate risks and opportunities & & C & & $\mathrm{C}$ & $\mathrm{I}$ & & & & & & & $\begin{array}{l}\mathrm{R} \\
\mathrm{A}\end{array}$ & $\mathrm{C}$ & & I \\
\hline Concept of operations & & & & $\begin{array}{l}\mathrm{R} \\
\mathrm{A}\end{array}$ & $\mathrm{C}$ & & & & $\mathrm{C}$ & & & & & & \\
\hline Configuration baselines & & $\mathrm{C}$ & & $\mathrm{C}$ & & & & & $\mathrm{C}$ & & $\mathrm{C}$ & & $\begin{array}{l}\mathrm{R} \\
\mathrm{A}\end{array}$ & & \\
\hline Configuration management record & & & & $\mathrm{C}$ & & & & & & & & & $\begin{array}{l}\mathrm{R} \\
\mathrm{A}\end{array}$ & & \\
\hline
\end{tabular}




\begin{tabular}{|c|c|c|c|c|c|c|c|c|c|c|c|c|c|c|c|}
\hline Input/Output & 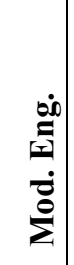 & $\begin{array}{l}\dot{j} \\
\dot{z} \\
\dot{\omega} \\
\dot{\omega}\end{array}$ & $\begin{array}{l}\dot{\dot{\vec{b}}} \\
\sum^{0} \\
\dot{\Xi} \\
\dot{\vec{\Omega}} \\
\dot{\vec{n}}\end{array}$ & $\begin{array}{l}\sum_{0}^{50} \\
\dot{\bar{g}} \\
\stackrel{0}{0}\end{array}$ & 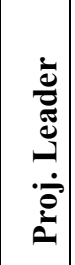 & 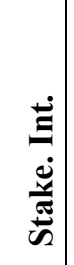 & 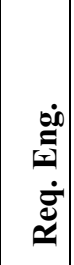 & 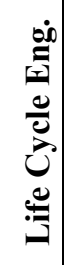 & $\begin{array}{l}\stackrel{\dot{\theta 0}}{\stackrel{0}{0}} \\
\dot{\underline{x}} \\
\dot{\vec{g}}\end{array}$ & 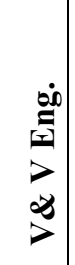 & 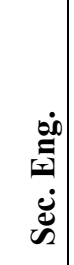 & 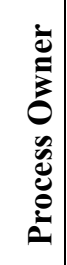 & 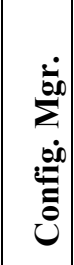 & $\begin{array}{l}\dot{\dot{g}} \\
\sum^{0} \\
\dot{\Xi} \\
\dot{\Xi} \\
\dot{\Xi}\end{array}$ & 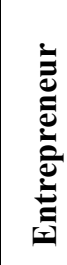 \\
\hline Configuration management report & & & & $\mathrm{C}$ & & & & & & & & & $\begin{array}{l}\mathrm{R} \\
\mathrm{A}\end{array}$ & & \\
\hline $\begin{array}{l}\text { Configuration management } \\
\text { strategy }\end{array}$ & & & & $\mathrm{C}$ & & & & & & & & & $\begin{array}{l}\mathrm{R} \\
\mathrm{A}\end{array}$ & & \\
\hline Customer satisfaction inputs & & $\mathrm{C}$ & $\mathrm{C}$ & & & $\begin{array}{l}\mathrm{R} \\
\mathrm{A}\end{array}$ & $\mathrm{C}$ & & & & & & & & \\
\hline Decision management strategy & & & & $\mathrm{C}$ & $\begin{array}{l}\mathrm{R} \\
\mathrm{A}\end{array}$ & & & & & & & & & & $\mathrm{C}$ \\
\hline Decision record & & & & $\mathrm{C}$ & $\begin{array}{l}\mathrm{R} \\
\mathrm{A}\end{array}$ & & & & & & & & & & $\mathrm{I}$ \\
\hline Decision report & & & & $\mathrm{C}$ & $\begin{array}{l}\mathrm{R} \\
\mathrm{A}\end{array}$ & & & & & & & & & & $\mathrm{I}$ \\
\hline Decision situation & & & & $\mathrm{C}$ & $\begin{array}{l}\mathrm{R} \\
\mathrm{A}\end{array}$ & & & & & & & & & & $\mathrm{I}$ \\
\hline Design definition record & A & & & & & $\mathrm{R}$ & & & & & & & & & \\
\hline Design definition strategy & $\begin{array}{l}\mathrm{R} \\
\mathrm{A}\end{array}$ & & & & & & & & & & & & & & \\
\hline Design traceability & $\begin{array}{l}\mathrm{R} \\
\mathrm{A}\end{array}$ & & & & & $\mathrm{C}$ & & & & & & & & & \\
\hline Disposal constraints & & & & & & & & $\begin{array}{l}\mathrm{R} \\
\mathrm{A}\end{array}$ & & & & & & & \\
\hline $\begin{array}{l}\text { Disposal enabling system } \\
\text { requirements }\end{array}$ & & & & & & & A & $\mathrm{R}$ & & & & & & & \\
\hline Disposal procedure & & & & $\mathrm{C}$ & & & & $\begin{array}{l}\mathrm{R} \\
\mathrm{A}\end{array}$ & & & & & & & \\
\hline Disposal record & & & & & & & & $\begin{array}{l}\mathrm{R} \\
\mathrm{A}\end{array}$ & & & & & & & \\
\hline Disposal report & & & & & & & & $\begin{array}{l}\mathrm{R} \\
\mathrm{A}\end{array}$ & & & & & & & \\
\hline Disposal strategy & & & & & & & & $\begin{array}{l}\mathrm{R} \\
\mathrm{A}\end{array}$ & & & & & & & \\
\hline Disposed system & & & & & & & & $\begin{array}{l}\mathrm{R} \\
\mathrm{A}\end{array}$ & & & & & & & \\
\hline Documentation tree & & & & $\begin{array}{l}\mathrm{R} \\
\mathrm{A}\end{array}$ & & & & & & & & & & $\mathrm{C}$ & \\
\hline Enabling system requirements & & & & & & & $\begin{array}{l}\mathrm{R} \\
\mathrm{A} \\
\end{array}$ & & & & & & & & \\
\hline Final RVTM & & $\mathrm{C}$ & $\mathrm{C}$ & $\mathrm{C}$ & & $\mathrm{C}$ & $\mathrm{A}$ & & & $\mathrm{R}$ & & & $\mathrm{C}$ & $\mathrm{C}$ & \\
\hline $\begin{array}{l}\text { Human resource management } \\
\text { plan }\end{array}$ & & & & & $\begin{array}{l}\mathrm{R} \\
\mathrm{A}\end{array}$ & & & & & & & $\mathrm{C}$ & & & $\mathrm{C}$ \\
\hline $\begin{array}{l}\text { Human resource management } \\
\text { record }\end{array}$ & & & & $\mathrm{R}$ & A & & & & & & & $\mathrm{C}$ & & & I \\
\hline $\begin{array}{l}\text { Human resource management } \\
\text { report }\end{array}$ & & & & $\mathrm{R}$ & A & & & & & & & $\mathrm{C}$ & & & I \\
\hline Implementation constraints & $\mathrm{C}$ & $\mathrm{A}$ & $\mathrm{C}$ & $\mathrm{I}$ & $\mathrm{I}$ & & & & $\mathrm{R}$ & & $\mathrm{C}$ & & $\mathrm{C}$ & & \\
\hline $\begin{array}{l}\text { Implementation enabling system } \\
\text { requirements }\end{array}$ & $\mathrm{C}$ & $\mathrm{A}$ & $\mathrm{C}$ & & & & $\mathrm{C}$ & & $\mathrm{R}$ & & $\mathrm{C}$ & & $\mathrm{C}$ & & \\
\hline Implementation record & & $\mathrm{A}$ & $\mathrm{C}$ & $\mathrm{I}$ & & & & & $\mathrm{R}$ & & & & & $\mathrm{C}$ & \\
\hline Implementation report & & $\mathrm{A}$ & $\mathrm{C}$ & $\mathrm{C}$ & & & & & $\mathrm{R}$ & & & & & $\mathrm{C}$ & \\
\hline
\end{tabular}




\begin{tabular}{|c|c|c|c|c|c|c|c|c|c|c|c|c|c|c|c|}
\hline Input/Output & 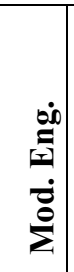 & $\begin{array}{l}\dot{j} \\
\dot{\vec{c}} \\
\dot{\omega} \\
\dot{\omega}\end{array}$ & 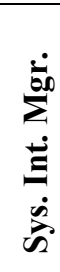 & 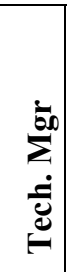 & 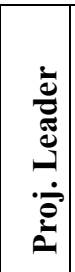 & 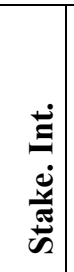 & 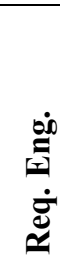 & $\begin{array}{l}\dot{00} \\
= \\
0 \\
0 \\
0 \\
0 \\
0 \\
0\end{array}$ & $\begin{array}{c}\dot{00} \\
\dot{=} \\
\dot{\mathbf{x}} \\
\dot{\mathbf{g}}\end{array}$ & 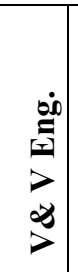 & 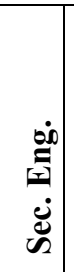 & 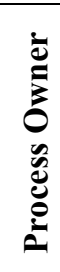 & 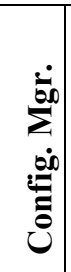 & $\begin{array}{c}\dot{\dot{b}} \\
\sum_{0} \\
\dot{\Xi} \\
\dot{\Xi} \\
\dot{\Xi}\end{array}$ & 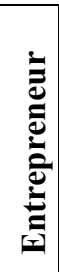 \\
\hline Implementation strategy & $\mathrm{C}$ & $\mathrm{A}$ & $\mathrm{C}$ & A & I & & $\mathrm{C}$ & & $\mathrm{R}$ & I & $\mathrm{C}$ & & $\mathrm{C}$ & & \\
\hline Implementation traceability & $\mathrm{C}$ & $\mathrm{A}$ & $\mathrm{C}$ & $\mathrm{C}$ & & & $\mathrm{C}$ & & $\mathrm{R}$ & & & & & & \\
\hline Information management record & & & & $\mathrm{C}$ & & & & & & & & & & $\begin{array}{l}\mathrm{R} \\
\mathrm{A}\end{array}$ & \\
\hline Information management report & & & & I & & & & & & & & & & $\begin{array}{l}\mathrm{R} \\
\mathrm{A}\end{array}$ & \\
\hline Information management strategy & & & & $\mathrm{C}$ & $\mathrm{I}$ & & & & & & & & & $\begin{array}{l}\mathrm{R} \\
\mathrm{A}\end{array}$ & \\
\hline Information repository & & & & & & & & & & & & & & $\begin{array}{l}\mathrm{R} \\
\mathrm{A}\end{array}$ & \\
\hline Infrastructure management plan & & & & $\begin{array}{l}\mathrm{R} \\
\mathrm{A}\end{array}$ & $\mathrm{C}$ & & & & & & & $\mathrm{C}$ & & & I \\
\hline Infrastructure management record & & & & $\begin{array}{l}\mathrm{R} \\
\mathrm{A}\end{array}$ & & & & & & & & $\mathrm{C}$ & & & \\
\hline Infrastructure management report & & & & $\begin{array}{l}\mathrm{R} \\
\mathrm{A}\end{array}$ & $\mathrm{I}$ & & & & & & & & & & \\
\hline Initial RVTM & & $\mathrm{C}$ & $\mathrm{C}$ & $\mathrm{C}$ & & $\mathrm{C}$ & $\mathrm{A}$ & & & $\mathrm{R}$ & & & $\mathrm{C}$ & $\mathrm{C}$ & \\
\hline Installation procedure & & & $\mathrm{C}$ & $\mathrm{A}$ & & & & & $\mathrm{R}$ & & $\mathrm{C}$ & & $\mathrm{C}$ & & \\
\hline Installed system & $\mathrm{C}$ & $\mathrm{C}$ & $\mathrm{C}$ & $\mathrm{C}$ & $\mathrm{C}$ & & & & $\mathrm{R}$ & & $\mathrm{C}$ & $\mathrm{C}$ & $\mathrm{C}$ & $\mathrm{C}$ & A \\
\hline $\begin{array}{l}\text { Integrated system or system } \\
\text { element }\end{array}$ & & $\mathrm{C}$ & $\mathrm{C}$ & $\mathrm{C}$ & & & $\mathrm{C}$ & & $\begin{array}{l}\mathrm{R} \\
\mathrm{A}\end{array}$ & $\mathrm{C}$ & $\mathrm{C}$ & & $\mathrm{C}$ & $\mathrm{C}$ & \\
\hline Integration constraints & $\mathrm{C}$ & $\mathrm{C}$ & & $\mathrm{C}$ & $\mathrm{I}$ & & $\mathrm{C}$ & & $\begin{array}{l}\mathrm{R} \\
\mathrm{A}\end{array}$ & & & & $\mathrm{C}$ & & \\
\hline $\begin{array}{l}\text { Integration enabling system } \\
\text { requirements }\end{array}$ & & & & & & & $\mathrm{C}$ & & $\begin{array}{l}\mathrm{R} \\
\mathrm{A}\end{array}$ & & & & & & \\
\hline Integration procedure & & & & $\mathrm{C}$ & & & & & $\begin{array}{l}\mathrm{R} \\
\mathrm{A}\end{array}$ & $\mathrm{C}$ & $\mathrm{C}$ & & $\mathrm{C}$ & $\mathrm{C}$ & \\
\hline Integration record & & & & & & & & & $\begin{array}{l}\mathrm{R} \\
\mathrm{A}\end{array}$ & & & & & & \\
\hline Integration report & & & & & & & & & $\begin{array}{l}\mathrm{R} \\
\mathrm{A}\end{array}$ & & & & & & \\
\hline Integration strategy & & & & $\mathrm{C}$ & $\mathrm{II}$ & & & & $\begin{array}{l}\mathrm{R} \\
\mathrm{A}\end{array}$ & & & & & & \\
\hline Interface definition & $\mathrm{C}$ & $\mathrm{C}$ & $\begin{array}{l}\mathrm{R} \\
\mathrm{A}\end{array}$ & I & & & $\mathrm{C}$ & & $\mathrm{C}$ & & $\mathrm{C}$ & & $\mathrm{C}$ & & \\
\hline $\begin{array}{l}\text { Interface definition update } \\
\text { identification }\end{array}$ & $\mathrm{C}$ & $\mathrm{C}$ & $\begin{array}{l}\mathrm{R} \\
\mathrm{A}\end{array}$ & I & & & $\mathrm{C}$ & & $\mathrm{C}$ & & $\mathrm{C}$ & & $\mathrm{C}$ & & \\
\hline Knowledge management plan & & & & $\begin{array}{l}\mathrm{R} \\
\mathrm{A}\end{array}$ & & & & & & & & & & & \\
\hline Knowledge management report & & & & $\begin{array}{l}\mathrm{R} \\
\mathrm{A}\end{array}$ & & & & & & & & & & & \\
\hline Knowledge management system & & & & $\begin{array}{l}\mathrm{R} \\
\mathrm{A}\end{array}$ & & & & & & & & & & & \\
\hline Life cycle concepts & & & $\mathrm{C}$ & I & I & & & $\begin{array}{l}\mathrm{R} \\
\mathrm{A}\end{array}$ & & & & & $\mathrm{C}$ & & $\mathrm{C}$ \\
\hline Life cycle constraints & & & & & & & & $\begin{array}{l}\mathrm{R} \\
\mathrm{A}\end{array}$ & & & & & $\mathrm{C}$ & & I \\
\hline $\begin{array}{l}\text { Life cycle model management } \\
\text { plan }\end{array}$ & & & $\mathrm{C}$ & I & I & & & $\begin{array}{l}\mathrm{R} \\
\mathrm{A}\end{array}$ & $\mathrm{C}$ & & & & & & \\
\hline
\end{tabular}




\begin{tabular}{|c|c|c|c|c|c|c|c|c|c|c|c|c|c|c|c|}
\hline Input/Output & 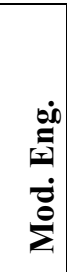 & 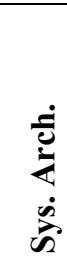 & $\begin{array}{l}\dot{\dot{\vec{b}}} \\
\sum^{0} \\
\dot{\Xi} \\
\dot{\vec{\Omega}} \\
\dot{\vec{n}}\end{array}$ & 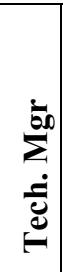 & 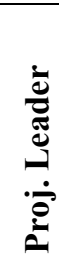 & 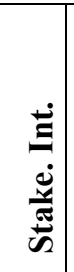 & 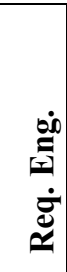 & 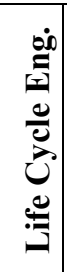 & $\begin{array}{l}\stackrel{\dot{00}}{\vec{y}} \\
\dot{\mathbf{g}} \\
\dot{\mathbf{g}}\end{array}$ & 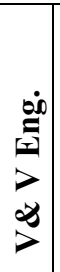 & 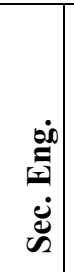 & 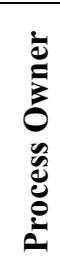 & 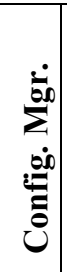 & 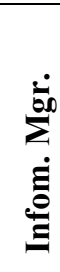 & 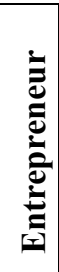 \\
\hline $\begin{array}{l}\text { Life cycle model management } \\
\text { record }\end{array}$ & & & & & & & & $\begin{array}{l}\mathrm{R} \\
\mathrm{A}\end{array}$ & & & & & & & \\
\hline $\begin{array}{l}\text { Life cycle model management } \\
\text { report }\end{array}$ & & & & $\mathrm{C}$ & $\mathrm{I}$ & & & $\begin{array}{l}\mathrm{R} \\
\mathrm{A}\end{array}$ & & & & & & & \\
\hline Life cycle models & $\mathrm{C}$ & $\mathrm{C}$ & $\mathrm{C}$ & $\mathrm{C}$ & & & & $\begin{array}{l}\mathrm{R} \\
\mathrm{A}\end{array}$ & $\mathrm{C}$ & & & & $\mathrm{C}$ & & \\
\hline Maintenance constraints & & & & A & $\mathrm{I}$ & & $\mathrm{C}$ & $\mathrm{R}$ & & & & & $\mathrm{C}$ & & $\mathrm{I}$ \\
\hline $\begin{array}{l}\text { Maintenance enabling system } \\
\text { requirements }\end{array}$ & C & C & & A & & & C & $\mathrm{R}$ & & & & & $\mathrm{C}$ & & \\
\hline Maintenance procedure & & & & A & & & $\mathrm{C}$ & $\mathrm{R}$ & & & & & $\mathrm{C}$ & & \\
\hline Maintenance record & & & & A & & & $\mathrm{C}$ & $\mathrm{R}$ & & & & & $\mathrm{C}$ & & \\
\hline Maintenance report & & & & A & & & $\mathrm{C}$ & $\mathrm{R}$ & & & & & $\mathrm{C}$ & & \\
\hline Maintenance strategy & & & & A & $\mathrm{C}$ & & $\mathrm{C}$ & $\mathrm{R}$ & & & & & $\mathrm{C}$ & & I \\
\hline Major stakeholder identification & & & & & I & $\begin{array}{l}\mathrm{R} \\
\mathrm{A}\end{array}$ & & & & & & & & & $\mathrm{C}$ \\
\hline Measurement data & & & $\mathrm{C}$ & & & & & & $\begin{array}{l}\mathrm{R} \\
\mathrm{A}\end{array}$ & & & & $\mathrm{C}$ & $\mathrm{C}$ & \\
\hline Measurement needs & & & $\mathrm{C}$ & & & & & & $\begin{array}{l}\mathrm{R} \\
\mathrm{A}\end{array}$ & & & & $\mathrm{C}$ & & \\
\hline Measurement record & & & $\mathrm{C}$ & & & & & & $\begin{array}{l}\mathrm{R} \\
\mathrm{A}\end{array}$ & & & & $\mathrm{C}$ & & \\
\hline Measurement report & & & $\mathrm{C}$ & & & & & & $\begin{array}{l}\mathrm{R} \\
\mathrm{A}\end{array}$ & & & & $\mathrm{C}$ & $\mathrm{I}$ & \\
\hline Measurement repository & & & $\mathrm{C}$ & & & & & & $\begin{array}{l}\mathrm{R} \\
\mathrm{A}\end{array}$ & & & & $\mathrm{C}$ & & \\
\hline Measurement strategy & & & $\mathrm{C}$ & & & & & & $\begin{array}{l}\mathrm{R} \\
\mathrm{A}\end{array}$ & & & & $\mathrm{C}$ & $\mathrm{C}$ & \\
\hline MOE data & & & $\mathrm{C}$ & & & & $\mathrm{C}$ & & $\mathrm{C}$ & & I & & & $\begin{array}{l}\mathrm{R} \\
\mathrm{A}\end{array}$ & \\
\hline MOE needs & & & $\mathrm{C}$ & & & & $\mathrm{C}$ & & $\mathrm{C}$ & & I & & & $\begin{array}{l}\mathrm{R} \\
\mathrm{A}\end{array}$ & \\
\hline MOP data & & & $\mathrm{C}$ & & & & $\mathrm{C}$ & & $\mathrm{C}$ & & I & & & $\begin{array}{l}\mathrm{R} \\
\mathrm{A}\end{array}$ & \\
\hline MOP needs & & & $\mathrm{C}$ & & & & $\mathrm{C}$ & & $\mathrm{C}$ & & $\mathrm{I}$ & & & $\begin{array}{l}\mathrm{R} \\
\mathrm{A}\end{array}$ & \\
\hline Operation constraints & & $\mathrm{C}$ & $\mathrm{C}$ & $\mathrm{C}$ & & & $\mathrm{C}$ & & $\begin{array}{l}\mathrm{R} \\
\mathrm{A}\end{array}$ & & & & $\mathrm{C}$ & & \\
\hline $\begin{array}{l}\text { Operation enabling system } \\
\text { requirements }\end{array}$ & & $\mathrm{C}$ & & $\mathrm{C}$ & & & $\mathrm{C}$ & & $\begin{array}{l}\mathrm{R} \\
\mathrm{A}\end{array}$ & & & & $\mathrm{C}$ & & \\
\hline Operation record & & & & $\mathrm{C}$ & & & & & $\begin{array}{l}\mathrm{R} \\
\mathrm{A}\end{array}$ & & & & $\mathrm{C}$ & & \\
\hline Operation report & & & & $\mathrm{C}$ & & & & & $\begin{array}{l}\mathrm{R} \\
\mathrm{A}\end{array}$ & & & & & & \\
\hline Operation strategy & & $\mathrm{C}$ & $\mathrm{C}$ & $\mathrm{A}$ & $\mathrm{C}$ & & & & $\mathrm{R}$ & & & & & & \\
\hline $\begin{array}{l}\text { Operator/maintainer training } \\
\text { materials }\end{array}$ & & & & & & & & & $\mathrm{C}$ & & & $\begin{array}{l}\mathrm{R} \\
\mathrm{A}\end{array}$ & & & \\
\hline Organization infrastructure & & & & & $\begin{array}{l}\mathrm{R} \\
\mathrm{A}\end{array}$ & & & & & & & $\mathrm{C}$ & & & $\mathrm{C}$ \\
\hline
\end{tabular}




\begin{tabular}{|c|c|c|c|c|c|c|c|c|c|c|c|c|c|c|c|}
\hline Input/Output & $\begin{array}{c}\dot{00} \\
\dot{=} \\
\dot{0} \\
\dot{E} \\
\dot{\Sigma}\end{array}$ & $\begin{array}{l}\dot{\bar{j}} \\
\dot{\vec{z}} \\
\dot{\dot{\omega}} \\
\dot{\omega}\end{array}$ & $\begin{array}{l}\dot{\dot{\theta 0}} \\
\sum \\
\dot{\Xi} \\
\dot{\Xi} \\
\dot{\infty}\end{array}$ & $\begin{array}{l}\sum_{0}^{50} \\
\dot{\bar{g}} \\
\stackrel{0}{0}\end{array}$ & 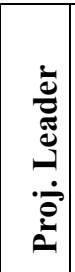 & 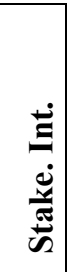 & 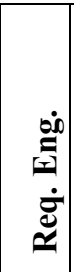 & 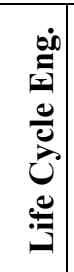 & 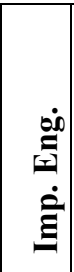 & 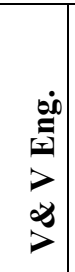 & 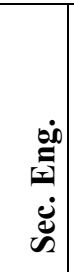 & 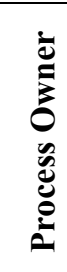 & 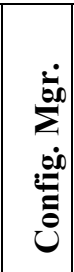 & 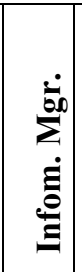 & 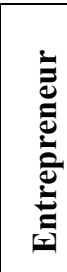 \\
\hline Organization infrastructure needs & & & & & $\begin{array}{l}\mathrm{R} \\
\mathrm{A}\end{array}$ & & & & & & & $\mathrm{C}$ & & & $\mathrm{C}$ \\
\hline Organization lessons learned & & & & & $\begin{array}{l}\mathrm{R} \\
\mathrm{A}\end{array}$ & & & & & & & $\mathrm{C}$ & & & C \\
\hline $\begin{array}{l}\text { Organization portfolio direction } \\
\text { and constraints }\end{array}$ & & & & & $\begin{array}{l}\mathrm{R} \\
\mathrm{A}\end{array}$ & & & & & & & $\mathrm{C}$ & & & $\mathrm{C}$ \\
\hline Organization strategic plan & & & & & $\begin{array}{l}\mathrm{R} \\
\mathrm{A}\end{array}$ & & & & & & & $\mathrm{C}$ & & & $\begin{array}{l}\mathrm{R} \\
\mathrm{A} \\
\end{array}$ \\
\hline Organization tailoring strategy & & & & & $\mathrm{C}$ & & & & & & & $\begin{array}{l}\mathrm{R} \\
\mathrm{A}\end{array}$ & & & \\
\hline $\begin{array}{l}\text { Organizational policies, } \\
\text { procedures, and assets }\end{array}$ & & & & & & & & & & & & & & & $\begin{array}{l}\mathrm{R} \\
\mathrm{A}\end{array}$ \\
\hline $\begin{array}{l}\text { Organizational process } \\
\text { performance measures data }\end{array}$ & & & & & $\mathrm{C}$ & & & & & & & & & $\mathrm{R}$ & $\mathrm{A}$ \\
\hline $\begin{array}{l}\text { Organizational process } \\
\text { performance measures needs }\end{array}$ & & & & & & & & & & & & & & & \\
\hline Portfolio management plan & & $\mathrm{C}$ & & $\mathrm{C}$ & $\mathrm{A}$ & $\mathrm{C}$ & & $\mathrm{C}$ & & & & & $\mathrm{C}$ & $\mathrm{I}$ & $\mathrm{R}$ \\
\hline Portfolio management record & & $\mathrm{C}$ & & $\mathrm{C}$ & A & $\mathrm{C}$ & & $\mathrm{C}$ & & & & & $\mathrm{C}$ & $\mathrm{I}$ & $\mathrm{R}$ \\
\hline Portfolio management report & & $\mathrm{C}$ & & $\mathrm{C}$ & $\mathrm{A}$ & $\mathrm{C}$ & & $\mathrm{C}$ & & & & & $\mathrm{C}$ & $\mathrm{I}$ & $\mathrm{R}$ \\
\hline Preliminary interface definition & & & & & & & & & & & & & & & \\
\hline Preliminary life cycle concepts & & & & & & & & & & & & & & & \\
\hline Preliminary MOE data & & & & & & & & & & & & & & & \\
\hline Preliminary MOE needs & & & & & & & & & & & & & & & \\
\hline Preliminary TPM data & & $\mathrm{C}$ & & $\mathrm{C}$ & & & & & $\mathrm{R}$ & $\mathrm{A}$ & & & $\mathrm{I}$ & & \\
\hline Preliminary TPM needs & & $\mathrm{C}$ & & $\mathrm{C}$ & & & & & $\mathrm{R}$ & $\mathrm{A}$ & & & & $\mathrm{C}$ & \\
\hline Preliminary validation criteria & & $\mathrm{C}$ & $\mathrm{I}$ & & & & $\mathrm{R}$ & & & A & & & & & \\
\hline Problem or opportunity statement & & $\mathrm{I}$ & & & $\begin{array}{l}\mathrm{R} \\
\mathrm{A}\end{array}$ & $\mathrm{C}$ & & $\mathrm{C}$ & & & & & & & $\mathrm{C}$ \\
\hline Procedures & $\mathrm{C}$ & $\mathrm{C}$ & $\mathrm{C}$ & & & $\mathrm{C}$ & $\mathrm{C}$ & $\mathrm{C}$ & $\mathrm{C}$ & $\mathrm{C}$ & $\mathrm{C}$ & $\begin{array}{l}\mathrm{R} \\
\mathrm{A}\end{array}$ & $\mathrm{C}$ & $\mathrm{C}$ & \\
\hline $\begin{array}{l}\text { Project assessment and control } \\
\text { record }\end{array}$ & & I & & I & $\begin{array}{l}\mathrm{R} \\
\mathrm{A}\end{array}$ & & & & & & & & & & $\mathrm{C}$ \\
\hline $\begin{array}{l}\text { Project assessment and control } \\
\text { strategy }\end{array}$ & & I & & I & $\begin{array}{l}\mathrm{R} \\
\mathrm{A}\end{array}$ & & & & & & & & & & $\mathrm{C}$ \\
\hline Project budget & $\mathrm{C}$ & & & $\mathrm{C}$ & $\begin{array}{l}\mathrm{R} \\
\mathrm{A}\end{array}$ & & $\mathrm{C}$ & $\mathrm{C}$ & & $\mathrm{C}$ & & & & & $\mathrm{C}$ \\
\hline Project change requests & & & & $\mathrm{C}$ & $\begin{array}{l}\mathrm{R} \\
\mathrm{A}\end{array}$ & $\mathrm{C}$ & $\mathrm{C}$ & $\mathrm{C}$ & & & & $\mathrm{C}$ & $\mathrm{C}$ & & \\
\hline Project constraints & & $\mathrm{C}$ & & $\mathrm{C}$ & $\begin{array}{l}\mathrm{R} \\
\mathrm{A}\end{array}$ & $\mathrm{C}$ & & & & & & & & & $\mathrm{C}$ \\
\hline Project control requests & & & & & $\begin{array}{l}\mathrm{R} \\
\mathrm{A}\end{array}$ & $\mathrm{C}$ & & & & & & & & & $\mathrm{C}$ \\
\hline Project direction & & & & & A & $\mathrm{C}$ & & $\mathrm{C}$ & & & & & & & $\mathrm{R}$ \\
\hline Project human resource needs & $\mathrm{C}$ & $\mathrm{C}$ & $\mathrm{C}$ & $\mathrm{C}$ & $\begin{array}{l}\mathrm{R} \\
\mathrm{A}\end{array}$ & $\mathrm{C}$ & $\mathrm{C}$ & $\mathrm{C}$ & $\mathrm{C}$ & $\mathrm{C}$ & $\mathrm{C}$ & $\mathrm{C}$ & $\mathrm{C}$ & $\mathrm{C}$ & $\mathrm{C}$ \\
\hline Project infrastructure & I & I & I & I & $\begin{array}{l}\mathrm{R} \\
\mathrm{A}\end{array}$ & I & $\mathrm{I}$ & I & I & I & I & I & I & I & I \\
\hline
\end{tabular}




\begin{tabular}{|c|c|c|c|c|c|c|c|c|c|c|c|c|c|c|c|}
\hline Input/Output & $\begin{array}{c}\dot{00} \\
\dot{=1} \\
\dot{0} \\
\dot{\theta} \\
\dot{\Sigma}\end{array}$ & 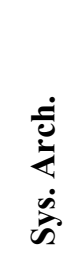 & $\begin{array}{l}\dot{\dot{b}} \\
\sum \\
\dot{\Xi} \\
\dot{\Xi} \\
\dot{\Delta} \\
\dot{\infty}\end{array}$ & 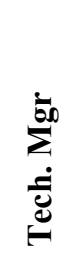 & 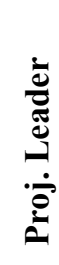 & 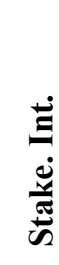 & 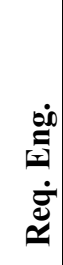 & 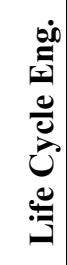 & 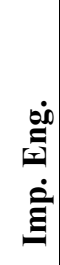 & 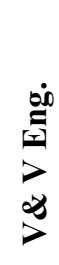 & $\begin{array}{l}\dot{000} \\
\dot{=1} \\
\dot{0} \\
\dot{\mathscr{D}}\end{array}$ & 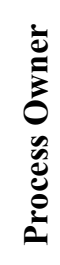 & 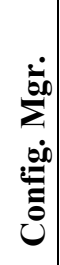 & 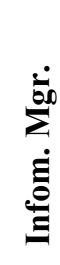 & 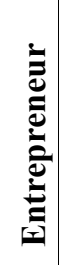 \\
\hline Project infrastructure needs & $\mathrm{C}$ & $\mathrm{C}$ & $\mathrm{C}$ & $\mathrm{C}$ & $\begin{array}{l}\mathrm{R} \\
\mathrm{A}\end{array}$ & $\mathrm{C}$ & $\mathrm{C}$ & $\mathrm{C}$ & $\mathrm{C}$ & $\mathrm{C}$ & $\mathrm{C}$ & $\mathrm{C}$ & $\mathrm{C}$ & $\mathrm{C}$ & $\mathrm{C}$ \\
\hline Project lessons learned & $\mathrm{C}$ & $\mathrm{C}$ & $\mathrm{C}$ & $\mathrm{C}$ & $\begin{array}{l}\mathrm{R} \\
\mathrm{A}\end{array}$ & $\mathrm{C}$ & $\mathrm{C}$ & $\mathrm{C}$ & $\mathrm{C}$ & $\mathrm{C}$ & $\mathrm{C}$ & $\mathrm{C}$ & $\mathrm{C}$ & $\mathrm{C}$ & $\mathrm{C}$ \\
\hline $\begin{array}{l}\text { Project performance measures } \\
\text { data }\end{array}$ & $\mathrm{C}$ & $\mathrm{C}$ & $\mathrm{C}$ & $\mathrm{C}$ & A & $\mathrm{C}$ & $\mathrm{C}$ & $\mathrm{C}$ & $\mathrm{C}$ & $\mathrm{C}$ & $\mathrm{C}$ & $\mathrm{R}$ & $\mathrm{C}$ & $\mathrm{C}$ & $\mathrm{C}$ \\
\hline $\begin{array}{l}\text { Project performance measures } \\
\text { needs }\end{array}$ & $\mathrm{C}$ & $\mathrm{C}$ & $\mathrm{C}$ & $\mathrm{C}$ & A & $\mathrm{C}$ & $\mathrm{C}$ & $\mathrm{C}$ & $\mathrm{C}$ & $\mathrm{C}$ & $\mathrm{C}$ & $\mathrm{R}$ & $\mathrm{C}$ & $\mathrm{C}$ & $\mathrm{C}$ \\
\hline Project planning record & & $\mathrm{C}$ & & $\mathrm{R}$ & A & & & & & & & & & & \\
\hline Project portfolio & & & & & $\begin{array}{l}\mathrm{R} \\
\mathrm{A}\end{array}$ & & & & & & & & & & $\mathrm{C}$ \\
\hline Project schedule & & & & & $\begin{array}{l}\mathrm{R} \\
\mathrm{A}\end{array}$ & & & & & & & & & & \\
\hline Project status report & & $\mathrm{C}$ & & & $\begin{array}{l}\mathrm{R} \\
\mathrm{A}\end{array}$ & & & & $\mathrm{C}$ & $\mathrm{C}$ & & $\mathrm{C}$ & $\mathrm{C}$ & $\mathrm{C}$ & \\
\hline Project tailoring strategy & & & & & $\begin{array}{l}\mathrm{R} \\
\mathrm{A}\end{array}$ & & & & & & & & & & $\mathrm{C}$ \\
\hline \multicolumn{16}{|l|}{$\begin{array}{l}\text { Quality management corrective } \\
\text { actions }\end{array}$} \\
\hline Qualified personnel & & & & & $\begin{array}{l}\mathrm{R} \\
\mathrm{A}\end{array}$ & & & & & & & & & & \\
\hline \multicolumn{16}{|l|}{$\begin{array}{l}\text { Quality assurance evaluation } \\
\text { report }\end{array}$} \\
\hline \multicolumn{16}{|l|}{ Quality assurance plan } \\
\hline \multicolumn{16}{|l|}{ Quality assurance record } \\
\hline \multicolumn{16}{|l|}{ Quality assurance report } \\
\hline \multicolumn{16}{|l|}{$\begin{array}{l}\text { Quality management evaluation } \\
\text { report }\end{array}$} \\
\hline \multicolumn{16}{|l|}{ Quality management guidelines } \\
\hline \multicolumn{16}{|l|}{ Quality management plan } \\
\hline \multicolumn{16}{|l|}{ Quality management record } \\
\hline \multicolumn{16}{|l|}{ Quality management report } \\
\hline \multicolumn{16}{|l|}{ Records } \\
\hline \multicolumn{16}{|l|}{ Reports } \\
\hline Request for supply & & $\mathrm{R}$ & $\mathrm{I}$ & $\mathrm{I}$ & A & $\mathrm{C}$ & $\mathrm{I}$ & $\mathrm{I}$ & & & & & $\mathrm{C}$ & & $\mathrm{C}$ \\
\hline Risk management strategy & & $\mathrm{C}$ & & $\mathrm{C}$ & $\mathrm{C}$ & $\mathrm{C}$ & & & & & $\mathrm{C}$ & $\begin{array}{l}\mathrm{R} \\
\mathrm{A}\end{array}$ & & & \\
\hline Risk record & & $\mathrm{C}$ & & $\mathrm{C}$ & $\mathrm{C}$ & $\mathrm{C}$ & & & & & $\mathrm{C}$ & $\begin{array}{l}\mathrm{R} \\
\mathrm{A}\end{array}$ & & & \\
\hline Risk report & & $\mathrm{C}$ & & $\mathrm{C}$ & $\mathrm{C}$ & $\mathrm{C}$ & & & & & $\mathrm{C}$ & $\begin{array}{l}\mathrm{R} \\
\mathrm{A}\end{array}$ & & & \\
\hline SEMP & & $\mathrm{C}$ & $\mathrm{C}$ & $\mathrm{R}$ & $\mathrm{I}$ & & & & $\mathrm{C}$ & $\mathrm{C}$ & & A & $\mathrm{C}$ & $\mathrm{C}$ & \\
\hline \multicolumn{16}{|l|}{ Source documents } \\
\hline Stakeholder needs & & & & & & $\begin{array}{l}\mathrm{R} \\
\mathrm{A}\end{array}$ & & & & & & & & & \\
\hline $\begin{array}{l}\text { Stakeholder needs and } \\
\text { requirements definition record }\end{array}$ & & & & & & $\mathrm{R}$ & A & & & & & $\mathrm{C}$ & & $\mathrm{C}$ & \\
\hline
\end{tabular}




\begin{tabular}{|c|c|c|c|c|c|c|c|c|c|c|c|c|c|c|c|}
\hline Input/Output & 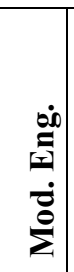 & $\begin{array}{l}\dot{\bar{e}} \\
\dot{\vec{\nu}} \\
\dot{\vec{\omega}}\end{array}$ & $\begin{array}{c}\dot{\Delta} \\
\sum_{0}^{0} \\
\dot{\Xi} \\
\dot{\Delta} \\
\dot{\Delta}\end{array}$ & 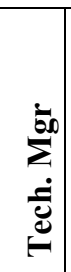 & 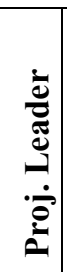 & 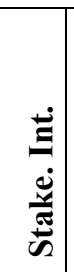 & 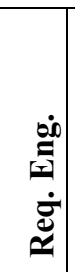 & $\begin{array}{l}\dot{00} \\
= \\
0 \\
0 \\
0 \\
0 \\
0 \\
.00 \\
:\end{array}$ & 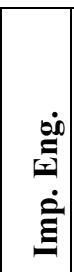 & $\begin{array}{c}\dot{\theta 0} \\
\stackrel{0}{=} \\
> \\
\ddot{z} \\
\dot{\gamma}\end{array}$ & $\begin{array}{l}\dot{00} \\
\text { ज्ञ } \\
\dot{\Xi} \\
\dot{\omega}\end{array}$ & 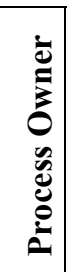 & 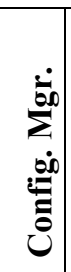 & $\begin{array}{l}\dot{\dot{b}} \\
\sum^{0} \\
\dot{\Xi} \\
\stackrel{\Xi}{\Xi}\end{array}$ & 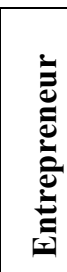 \\
\hline $\begin{array}{l}\text { Stakeholder needs and } \\
\text { requirements definition strategy }\end{array}$ & & & & & & $\mathrm{R}$ & A & & & & & & & & \\
\hline Stakeholder requirements & & & & & & $\mathrm{R}$ & $\mathrm{A}$ & & & & & & & & \\
\hline $\begin{array}{l}\text { Stakeholder requirements } \\
\text { traceability }\end{array}$ & & & & & & $\mathrm{R}$ & $\mathrm{A}$ & & $\mathrm{C}$ & $\mathrm{C}$ & & & & $\mathrm{C}$ & \\
\hline Strategy documents & & & & $\mathrm{C}$ & & $\begin{array}{l}\mathrm{R} \\
\mathrm{A}\end{array}$ & & $\mathrm{C}$ & & & & & & & $\mathrm{C}$ \\
\hline Supplied system & & $\mathrm{R}$ & & $\mathrm{A}$ & & & $\mathrm{C}$ & & $\mathrm{C}$ & & & & $\mathrm{C}$ & & \\
\hline Supply agreement & & & & $\mathrm{A}$ & $\mathrm{R}$ & & $\mathrm{C}$ & & & & & & & & \\
\hline Supply payment & & & & $\mathrm{C}$ & $\begin{array}{l}\mathrm{R} \\
\mathrm{A}\end{array}$ & & & & & & & & & & \\
\hline Supply record & & $\mathrm{R}$ & & $\mathrm{A}$ & $\mathrm{C}$ & & & & & & & & & & \\
\hline Supply report & & $\mathrm{R}$ & & $\mathrm{A}$ & $\mathrm{C}$ & & & & & & & & & & \\
\hline Supply response & & & & $\mathrm{A}$ & $\mathrm{R}$ & & & & & & & & & & \\
\hline Supply strategy & & $\mathrm{R}$ & I & I & A & $\mathrm{C}$ & I & I & & & & & $\mathrm{C}$ & & $\mathrm{C}$ \\
\hline System analysis record & $\mathrm{C}$ & $\mathrm{C}$ & & $\mathrm{C}$ & & & $\mathrm{C}$ & & $\mathrm{C}$ & $\mathrm{C}$ & $\mathrm{C}$ & $\mathrm{A}$ & & $\mathrm{R}$ & \\
\hline System analysis report & $\mathrm{C}$ & $\mathrm{C}$ & & $\mathrm{C}$ & & & $\mathrm{C}$ & & $\mathrm{C}$ & $\mathrm{C}$ & $\mathrm{C}$ & $\mathrm{A}$ & & $\mathrm{R}$ & \\
\hline System analysis strategy & & & & $\mathrm{C}$ & & & & & & & & $\begin{array}{l}\mathrm{R} \\
\mathrm{A}\end{array}$ & & $\mathrm{C}$ & \\
\hline System architecture description & $\mathrm{C}$ & $\mathrm{A}$ & & I & I & & $\mathrm{C}$ & & $\mathrm{R}$ & & & & $\mathrm{C}$ & & \\
\hline System architecture rationale & $\mathrm{C}$ & $\mathrm{A}$ & & $\mathrm{I}$ & I & & $\mathrm{C}$ & & $\mathrm{R}$ & & & & $\mathrm{C}$ & $\mathrm{C}$ & \\
\hline System design description & $\mathrm{C}$ & $\mathrm{A}$ & $\mathrm{C}$ & $\mathrm{I}$ & $\mathrm{I}$ & $\mathrm{C}$ & $\mathrm{C}$ & & $\mathrm{R}$ & & & & $\mathrm{C}$ & $\mathrm{C}$ & \\
\hline System design rationale & $\mathrm{C}$ & $\mathrm{A}$ & $\mathrm{C}$ & I & I & $\mathrm{C}$ & $\mathrm{C}$ & & $\mathrm{R}$ & & & & $\mathrm{C}$ & $\mathrm{C}$ & \\
\hline System element descriptions & & $\mathrm{A}$ & $\mathrm{C}$ & $\mathrm{I}$ & & & $\mathrm{C}$ & & $\mathrm{R}$ & & & & $\mathrm{C}$ & & \\
\hline System element documentation & $\mathrm{C}$ & $\mathrm{C}$ & & $\mathrm{C}$ & & & & & $\mathrm{R}$ & & & & & $\mathrm{A}$ & \\
\hline System elements & & $\mathrm{A}$ & & $\mathrm{R}$ & & & & & $\mathrm{R}$ & & & & $\mathrm{I}$ & & \\
\hline System function definition & & $\begin{array}{l}\mathrm{R} \\
\mathrm{A}\end{array}$ & $\mathrm{C}$ & & & & $\mathrm{C}$ & & & & & & & & \\
\hline System function identification & & $\begin{array}{l}\mathrm{R} \\
\mathrm{A}\end{array}$ & $\mathrm{C}$ & & & & $\mathrm{C}$ & $\mathrm{C}$ & & & & & $\mathrm{C}$ & & $\mathrm{C}$ \\
\hline $\begin{array}{l}\text { System functional interface } \\
\text { identification }\end{array}$ & & $\mathrm{C}$ & $\begin{array}{l}\mathrm{R} \\
\mathrm{A}\end{array}$ & & & & & & $\mathrm{C}$ & & & & & & \\
\hline System requirements & & $\mathrm{C}$ & $\mathrm{C}$ & & & $\mathrm{C}$ & $\begin{array}{l}\mathrm{R} \\
\mathrm{A}\end{array}$ & & & & & & $\mathrm{C}$ & $\mathrm{C}$ & \\
\hline $\begin{array}{l}\text { System requirements definition } \\
\text { record }\end{array}$ & & & & & & & $\begin{array}{l}\mathrm{R} \\
\mathrm{A}\end{array}$ & & & & & & & & \\
\hline $\begin{array}{l}\text { System requirements definition } \\
\text { strategy }\end{array}$ & & $\mathrm{C}$ & & $\mathrm{R}$ & & $\mathrm{C}$ & $\mathrm{A}$ & $\mathrm{C}$ & & $\mathrm{C}$ & & $\mathrm{C}$ & & & $\mathrm{C}$ \\
\hline System requirements traceability & & $\mathrm{R}$ & & & & & A & & $\mathrm{C}$ & $\mathrm{C}$ & & & & & \\
\hline TPM data & & $\mathrm{C}$ & & $\mathrm{C}$ & & & & & $\mathrm{R}$ & $\mathrm{A}$ & & & I & & \\
\hline TPM needs & & $\mathrm{C}$ & & $\mathrm{C}$ & & & & & $\mathrm{R}$ & $\mathrm{A}$ & & & & $\mathrm{C}$ & \\
\hline \multicolumn{16}{|l|}{ Trained operators and maintainers } \\
\hline Transition constraints & & $\mathrm{C}$ & & $\mathrm{R}$ & $\mathrm{C}$ & & & $\mathrm{C}$ & & & & A & & & \\
\hline $\begin{array}{l}\text { Transition enabling system } \\
\text { requirements }\end{array}$ & & $\mathrm{C}$ & & $\mathrm{R}$ & $\mathrm{C}$ & & & $\mathrm{C}$ & & $\mathrm{C}$ & & $\mathrm{A}$ & & & \\
\hline Transition record & & $\mathrm{C}$ & & $\mathrm{C}$ & & & & $\mathrm{R}$ & & & & & & $\mathrm{C}$ & \\
\hline Transition report & & $\mathrm{C}$ & & $\mathrm{C}$ & & & & $\mathrm{R}$ & & & & & & $\mathrm{C}$ & \\
\hline
\end{tabular}




\begin{tabular}{|c|c|c|c|c|c|c|c|c|c|c|c|c|c|c|c|}
\hline Input/Output & $\begin{array}{l}\stackrel{\dot{0} 0}{=} \\
\dot{\mid c} \\
\dot{0} \\
\dot{0}\end{array}$ & $\begin{array}{l}\dot{e} \\
\dot{\vec{z}} \\
\dot{\dot{d}} \\
\dot{\omega}\end{array}$ & $\begin{array}{c}\dot{\dot{\omega}} \\
\sum^{0} \\
\dot{\dot{\Xi}} \\
\dot{\dot{\omega}} \\
\dot{\omega}\end{array}$ & 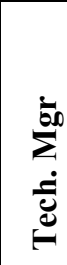 & 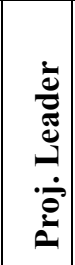 & 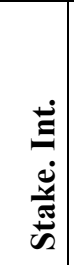 & 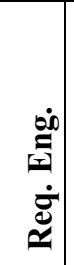 & 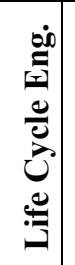 & $\begin{array}{l}\dot{0} 0 \\
\dot{\mid=} \\
\dot{\mathbf{g}} \\
\dot{\Xi}\end{array}$ & 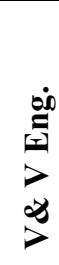 & 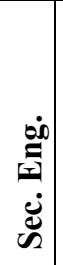 & 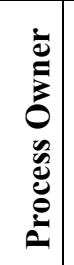 & 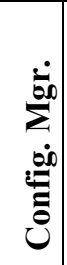 & 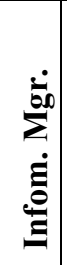 & 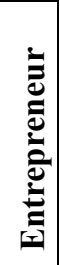 \\
\hline Transition strategy & & $\mathrm{C}$ & & $\mathrm{C}$ & $\mathrm{C}$ & & & $\mathrm{C}$ & & & & $\begin{array}{l}\mathrm{R} \\
\mathrm{A}\end{array}$ & & & \\
\hline Updated RVTM & & $\mathrm{C}$ & $\mathrm{C}$ & $\mathrm{C}$ & & $\mathrm{C}$ & $\mathrm{A}$ & & & $\mathrm{R}$ & & & $\mathrm{C}$ & $\mathrm{C}$ & \\
\hline Validated requirements & & $\mathrm{C}$ & & & & $\mathrm{C}$ & $\begin{array}{l}\mathrm{R} \\
\mathrm{A}\end{array}$ & & & $\mathrm{C}$ & & & & & \\
\hline Validated system & $\mathrm{C}$ & $\mathrm{C}$ & & & & & $\mathrm{C}$ & & & $\begin{array}{l}\mathrm{R} \\
\mathrm{A}\end{array}$ & & & & & \\
\hline Validation constraints & & $\mathrm{C}$ & & & & & $\mathrm{C}$ & & & $\begin{array}{l}\mathrm{R} \\
\mathrm{A}\end{array}$ & & $\mathrm{C}$ & & & \\
\hline Validation criteria & & $\mathrm{C}$ & I & & & & $\mathrm{R}$ & & & $\mathrm{A}$ & & & & & \\
\hline $\begin{array}{l}\text { Validation enabling system } \\
\text { requirements }\end{array}$ & & & & & & & & & & $\mathrm{R}$ & & A & & $\mathrm{C}$ & \\
\hline Validation procedure & & $\mathrm{C}$ & & $\mathrm{C}$ & $\mathrm{I}$ & & & & & $\begin{array}{l}\mathrm{R} \\
\mathrm{A}\end{array}$ & & $\mathrm{C}$ & & & \\
\hline Validation record & & $\mathrm{C}$ & & $\mathrm{C}$ & $\mathrm{I}$ & & & & & $\begin{array}{l}\mathrm{R} \\
\mathrm{A}\end{array}$ & & & & $\mathrm{C}$ & \\
\hline Validation report & & & & & & & & & & $\begin{array}{l}\mathrm{R} \\
\mathrm{A}\end{array}$ & & & & $\mathrm{C}$ & \\
\hline Validation strategy & & $\mathrm{C}$ & & $\mathrm{C}$ & & & & & & $\begin{array}{l}\mathrm{R} \\
\mathrm{A}\end{array}$ & & $\mathrm{C}$ & & $\mathrm{C}$ & \\
\hline Verification constraints & & $\mathrm{C}$ & & & & & $\mathrm{C}$ & & & $\begin{array}{l}\mathrm{R} \\
\mathrm{A}\end{array}$ & & $\mathrm{C}$ & & & \\
\hline Verification criteria & & $\mathrm{C}$ & $\mathrm{I}$ & & & & $\mathrm{R}$ & & & A & & & & & \\
\hline $\begin{array}{l}\text { Verification enabling system } \\
\text { requirements }\end{array}$ & & & & & & & & & & $\mathrm{R}$ & & A & & $\mathrm{C}$ & \\
\hline Verification procedure & & $\mathrm{C}$ & & $\mathrm{C}$ & $\mathrm{I}$ & & & & & $\begin{array}{l}\mathrm{R} \\
\mathrm{A}\end{array}$ & & $\mathrm{C}$ & & & \\
\hline Verification record & & $\mathrm{C}$ & & $\mathrm{C}$ & $\mathrm{I}$ & & & & & $\begin{array}{l}\mathrm{R} \\
\mathrm{A}\end{array}$ & & & & $\mathrm{C}$ & \\
\hline Verification report & & & & & & & & & & $\begin{array}{l}\mathrm{R} \\
\mathrm{A}\end{array}$ & & & & $\mathrm{C}$ & \\
\hline Verification strategy & & $\mathrm{C}$ & & $\mathrm{C}$ & & & & & & $\begin{array}{l}\mathrm{R} \\
\mathrm{A}\end{array}$ & & $\mathrm{C}$ & & $\mathrm{C}$ & \\
\hline Verified system & $\mathrm{C}$ & $\mathrm{C}$ & & & & & $\mathrm{C}$ & & & $\begin{array}{l}\mathrm{R} \\
\mathrm{A}\end{array}$ & & & & & \\
\hline WBS & & & & $\mathrm{R}$ & C & & & & & & & A & & & \\
\hline
\end{tabular}

